\title{
The Direction of Drilling Wells as a Factor of Improving the Crushing of Sedimentary
}

\author{
Igor Katanov ${ }^{1, *}$, Andrey Sysoev ${ }^{1}$, and Ivan Panachev ${ }^{1}$ \\ ${ }^{1}$ T.F. Gorbachev Kuzbass State Technical University, 650000, Kemerovo, Russian Federation
}

\begin{abstract}
The task of the work is to improve the quality of preparation of the rock mass for excavation. Explosive destruction of the rock is based on several theories developed by well-known scientists. The improvement of quality of preparation of rock mass to excavation without an increase in the value of specific consumption of explosives is important in the present time. The analysis of scientific works and industrial results of explosive preparation of the overburden removal is made. It is established that depending on the angle of the explosive charges meeting with the rock layers, the quality of its crushing changes. Modern drilling machines can drill at any angle. It is proposed to drill wells perpendicular to the rock layers. The proposal differs in originality from the previously known principles of charge placement of explosives in wells. This method of charge placement has a positive effect in sedimentary rocks in coal mines. The results of industrial explosions in coal mines have confirmed the theoretical reasoning.
\end{abstract}

\section{Introduction}

Mining and geological conditions in the Kuznetsk coal basin are diverse and complex. The layers are deposited in the form of the set of rocks from the gentle to the steep drop. Overburden rocks of coal deposits of Kuzbass are represented by sandstones, siltstones and mudstones. The structure of rock masses, sometimes even within the same blasting block changes from small-block structure (with dimensions of natural separateness of 200-400 $\mathrm{mm}$ ) to large-block structure (with sizes 3000-5000 mm). According to the degree of explosion, overburden rocks are divided into three categories: highly explosive rocks, medium explosive rocks and hard-to-break rocks. In some cases, the strong crushing of rocks, particularly of large-block-grained structure in the existing technology is not enough to ensure productive operation of mining and transport equipment.

One of the important directions of improvement of explosive works is increase of explosion efficiency as a result of rational redistribution of energy of explosive substance. The use of modern explosives by traditional methods of blasting over crushing is accompanied by a significant rock milling in the near-to-charge zone. This zone is at the same time an area of strong energy absorption. Large energy loss at the initial stage of development of the explosion cannot be subsequently compensated.

\footnotetext{
*Corresponding author: noa-0025@yandex.ru
} 
The transfer of energy in the explosion of a solid charge takes place almost immediately. By changing the spatial arrangement of borehole charges in the rock mass, it is possible to distribute rationally the explosion energy in the rock mass.

The effect is achieved, firstly, if rock fragmentation occurs when the external load creates an appropriate limiting tension. When crushing rocks, it is necessary that the main compression wave had an amplitude above the rock strength limit and secondly, if the explosion of the borehole charge takes into account the relationship of shear force stresses in the direction of the lamination and the direction of the fracturing of the rock mass.

The first attempt to describe the process of rock mass destruction under the action of explosion in case of inhomogeneity of the structure occurs in the following works [1,2]. Proceeding from the fundamental provisions of the energy of the explosion theory M. V. Machinskiy explains the occurrence of cracks in weak areas of the blasted rock mass as a result of the action of stress wave, after passing which, the cracks continue to grow and at some point in time merge, as a result of it, the rock is fragmented.

Well-reasonable representations of the qualitative picture of the explosion effect on the environment are presented from the point of view of physics in the works of G. I. Pokrovskiy [3, 4], who considers the position of self-similarity of shock waves propagation in explosion and the main energy dependences, establishes a number of parameters of the shock wave arising in the mass during the explosion of a spherical charge of the explosive substance. Thus, the maximum value of the excess pressure of the compression wave in a solid mass and the duration of the excess positive pressure in the compression wave are determined.

M. A. Cook [5] indicates the existence of three basic theories of explosive destruction of rocks by explosion. The first theory is shock-wave theory of rock blasting or funnel formation. The main elements of shock-wave theory of rock blasting are the impedances of explosives and rocks, which determine the role of reflected waves in the mechanism of destruction $[6,7]$.

The theory of radial cracking, developed by a group of Swedish scientists, in which the determining role in the mechanism of destruction is given to the wave of displacement at an early stage of shock wave propagation and the subsequent development of radial cracks. M. A. Cook believes that this theory is also a shock-wave theory, although the role of rock breaking at the free surface in it is reduced to a minimum. The third theory is known as energy one $[8,9]$. According to this theory, the pressure of detonation products compresses the explosive rock mass surface, bringing it into a tense state, facilitating subsequent crushing, and creating a velocity territory in the rock mass, conducive to the beginning of the final stage of primary crushing.

The main prerequisites of O. E. Vlasov's hydrodynamic hypothesis are: incompressibility of the rock mass, representation of the rock mass as an ideal fluid, instantaneous propagation of explosion energy in the rock mass. Assuming that the energy of the explosion is transferred to the rock mass as kinetic energy, the motion of the incompressible rock mass is expressed in the form of known equations of hydrodynamics of the ideal fluid [10-12].

In the matter of destruction of the rock mass by explosion A. N. Hanukayev [13] follows the theory that the process of destruction occurs under the action of elastic waves. Based on the theory of rock fragmentation by the reflected wave, a position in which the process of destruction of rocks largely depends on their acoustic rigidity is put forward by the scientists.

E. P. Maksimova [14], conducting experimental explosions on concrete cubes, came to the conclusion that the bulk of the destruction is caused by cracks extending from the charging chamber. The amount of destruction caused by reflected waves is insignificant. 
The generalized F. A. Baum's ideas about the mechanism of rock fragmentation by explosion, apparently, most fully reflect the essential aspects of this process occurring under the combined influence of detonation products, shock waves and discharge waves. In time and by the nature of the physical phenomena that determine it, the explosion is characterized by several successive stages $[15,16]$. At the initial stage of the process, the main role is played by expanding detonation products, which at the first moment are under pressure of about $105 \mathrm{~kg} / \mathrm{cm} 2$. The expansion of detonation products proceeds isentropicfirst by law, and then at lower pressures - by law .At this stage of development of the explosion in the rock mass range plastic flow of the rocks, the occurrence of radial cracking and fragmentation occurs. On contact of the charge-rock, the law of compressibility which determines the initial parameters and the number of patterns of distribution of shock waves takes place [17]. The second stage of the process begins after the shock waves come on the free surface. It is associated with the propagation of the reflected wave from this surface of the discharge and its interaction with the tail part of the traveling compression wave. As a result of this process in the rock leads to significant tensile stresses and ultimately cracking new ones. The intersection of these cracks and natural micro-and macro-cracks, available in the rock, with a system of radial cracks, arising in it at the previous stage of the explosion, leads to the formation of a spatial mesh of cracks, the nature of which to some extent determines the possible size and the number of fragmented during the explosion of rock pieces. The role of cracking phenomena in the process of crushing of rocks by explosion is very small. The third and the final stage of the process leads mainly to the influence on the rock which has already expanded to detonation products. By the time of its full expansion, detonation products still contain about $50 \%$ of the explosion energy from its total amount. Under the influence of this energy, there are different types of rock destruction with the penetration of gases into the cracks, separation of individual pieces from the rock mass, etc., the movement of the destroyed rock mass and the expansion of individual pieces of the mass.

A. F. Belyaev and M. A. Sadovskiy [18], considering the nature of the blasting and high-explosive action, divide the various forms of destruction of the solid rock mass into two types. The first type is the destruction of the rock mass in the immediate proximity of the charge, manifested in the re-grinding of the rock mass by the frontal part of the explosion pulse. The second type is the destruction of the rock mass outside the zone of brisant action, manifested in fracturing and displacement due to the main part of the explosion wave. The authors note that the increase in useful work can be achieved by a more favorable redistribution of the full work of the explosion in certain forms, as well as reducing losses. An increase in the useful forms of work can be achieved, reducing blasting effects of the explosion and increasing explosive action.

In F. I. Kucheryavyi, M. G. Novozhilov and M. F. Drukovannyi' s works [19], it is experimentally proved that the intensity of rock crushing along with the amount of pressure at the wave front is significantly influenced by the time of the explosion on the rock mass G. P. Paramonov [20] shows that the failure mechanism of fractured mass depends on what the cracks are filled with. If the cracks are filled with solid material, the destruction is mainly due to the waves of stresses. When there is air in the cracks, the wave of stresses destroys only the separateness in which the charge is, further destruction occurs due to detonation products.

\section{Results}

For most rocks, the tensile strength of uniaxial compression is higher than the tensile strength. Roughly can be to accept [21]. When large differences in rock strength for uniaxial compression and extension, the region of possible angles for which destruction will 
occur in the perpendicular to the plane of the layer (interlayer) direction, and not along it, very limited. Most rock masses are heterogeneous and anisotropic due to geological reasons, i.e. they have several crack systems. The causes of anisotropy are layering, fracturing, different strength of the minerals that make up rocks. Sedimentary rocks are characterized by the presence of three crack systems. Cracks separating the layers (layers) and two mutually perpendicular cutting systems of cracks, which determine the block structure of the array.

The shape of the zone of controlled crushing in the cross-section perpendicular to the axis of the borehole charge is determined by the degree of anisotropy of the rock mass. In general, in this section the curve of the fracture anisotropy of the rock mass will be close to the shape of the ellipse. Inversely proportional to the curve of the anisotropy of the fracture and the speed of longitudinal waves in the mass will change.

Now the method of explosive preparation of rock mass for excavating by the method of borehole charges of rocks with layered structure, includes drilling of parallel rows of vertical or inclined wells, charging them with explosive substance with the subsequent slaughter, installation of an explosive chain and short-delay blasting.

Wells are placed from each other at a calculated distance on a rectangular or checkerboard grid. Moreover, wells in the rock mass are arranged so that the longitudinal axis of each well on the plane of the slope of the ledge is designed as a perpendicular to the upper and lower edges of the ledge. In this case, the axis of the well crosses the plane of layers at some angle (Fig. 1). The most favorable conditions for crushing the rock mass in most cases occur when the fragmentation load in the layer is strictly perpendicular to the deposition (Fig. 1b) or at spreading of layers of the parallel line of a slope of a ledge (Fig. 1 e). If the angle of incidence of layers with the axis of the well is more than 35 degrees. (Fig. $1 \mathrm{c}, \mathrm{f}$ ) attenuation of the voltage wave and deterioration of the quality of crushing rock mass can be possible.

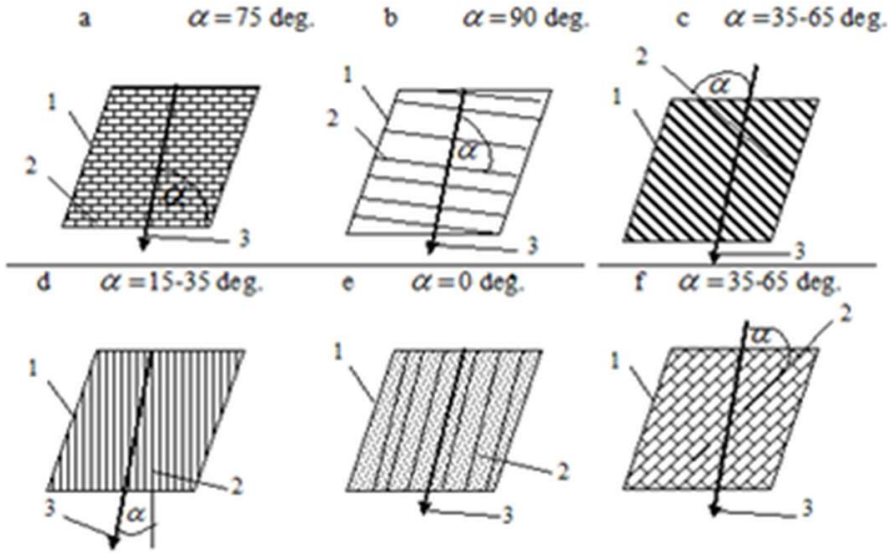

Fig. 1. The scheme of spatial placement of wells in the rock mass comparatively to bedding: 1-slope of the ledge; 2-bedding of the rock; 3 - the direction of drilling.

The design of explosions does not take into account the relationship of shear stresses in the direction of the layered and fractured direction of the rock mass. If the angle of incidence of layers with the axis of the well is more than 35 degrees. the attenuation of a wave of pressure within the individual, and deterioration of the quality of fragmentation of the rock mass may take place. Evaluation of the surface collapse and the bottom of the excavator by a photopolarimetric method allowed to judge about relatively non-uniform crushing of the rock. Separate pieces of rock were observed in the form of parallelepipeds more than $2 \mathrm{~m}$ long, $0.7 \mathrm{~m}$ wide and $0.7 \mathrm{~m}$ thick. 
Therefore, for uniform crushing of the rock mass at an unfavorable location of the charge relative to the deposition, an increased specific consumption of the explosive is necessary. If you use charges with the same specific consumption of explosives without taking into account the structure of the rock mass, you can get unpredictable results.

The analysis of the quality of explosive rock preparation for excavation in the coal mine "Zarechnyi" in Kuzbass made it possible to obtain a pattern of changing the size of the piece in the collapse, depending on the spatial location of wells in the layered rock mass (Fig. 2).

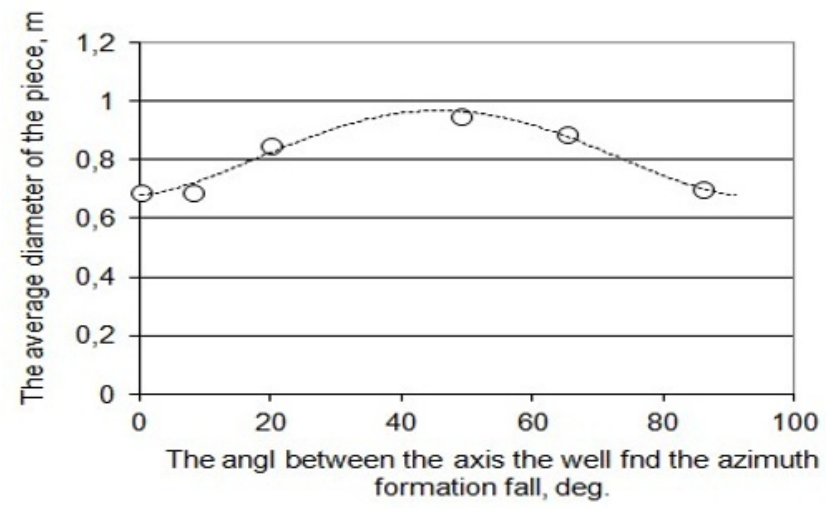

Fig. 2. Changing the size of the piece in the destruction, depending on the angle of the meeting of the borehole charge with the layers of the rock mass.

The analysis of the graph shows that the good quality of the preparation of the overburden removing to the excavation is obtained when drilling wells comparatively to the plane of deposition at an angle of 85-90 degrees and in case of coincidence of the direction of drilling with the plane of layers.

\section{Discussion}

Therefore, for uniform crushing of the rock mass at an unfavorable location of the charge relative to the deposition, an increased specific consumption of the explosive is necessary. If you use charges with the same specific consumption of explosives without taking into account the structure of the rock mass, you can get unpredictable results.

The analysis of the graph shows that the good quality of the preparation of the overburden removing to the exavation is obtained when drilling wells comparatively the plane of deposition at an angle of 85-90 degrees and in case of coincidence of the direction of drilling with the plane of layers.

Therefore, in order to obtain uniform crushing of the rock within the entire ledge before the design of drilling and blasting operations, an examination of the mass with a georadar "OKO-2" with a screened separate antenna unit "AB-90" was carried out. The analysis of radargrams and photos of the slope of the ledge in the direction from West to East showed that the angle of incidence of the surfaces of layers is 10-22 degrees, and azimuth of their fall is 120-140 degrees. Then the surfaces of the layers are discharged. After $85-100 \mathrm{~m}$ the layers change the angle of incidence to 146 degrees (Fig. 3).

Blasting of the rock mass with a volume of $136908 \mathrm{~m} 3$ was carried out in the face of the excavator PH-2300 № 2387 with a bucket capacity of $25 \mathrm{~m} 3$ on the horizon +279 . The height of the ledge was $15 \mathrm{~m}$. the diameter of the wells is $0.2 \mathrm{~m} ., 0,2 \mathrm{M}$. the rocks are represented by sandstone, which belongs to the medium-fractured rocks. The strength coefficient according to Professor M. M. Protodiakonov is $f=5,5$. The average diameter of natural 
separateness is $1.1 \mathrm{~m}$. The grid of the wells is rectangular and the size is $6 \square 6 \mathrm{~m}$. Since the Federal regulations and safety rules currently allow the drilling rig to be installed at any angle in relation to the slope of the ledge, in the Western part of the well block it was drilled at an angle of 75 degrees. to the horizon at the same time, the projection of the axis of the drilling stake on the plane of the layers was close to the normal comparatively to the azimuth of the stretch of the layers (Fig. 3a). The middle part of the block was trimmed according to the usual scheme, when wells were drilled at an angle of 75 degrees. to the horizon, and the projection of the longitudinal axis of the drill was perpendicular to the slope of the ledge (Fig. 3b). In the Eastern part of the block wells were drilled at an angle of 75 degrees. at the same time, the projection of the axis of the drilling stake on the plane of the layers was close to perpendicular to the azimuth of the fall of the layers (Fig. 3c).
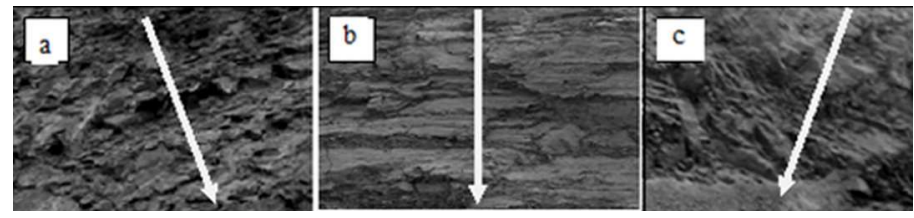

Fig. 3. Kinematic scheme of operation of hydraulic backhoe CAT $330 \mathrm{~S}$ at a layer height $\mathrm{hl}=5.0 \mathrm{~m}$ and the dip angle of coal seam $\varphi=25^{\circ}$.

The wells were charged with combined charges of granulites and emulsoid RD A-20, dispersed in height. The dispersal of borehole charges was carried out with the sleeve-350250-7. The scheme of blasting was diagonal one with use of a system of initiation with shock-wave tubes for installation of a surface rionel-X network (42, $67 \mathrm{MS})$ and downhole Rionel-MS-20.

The explosion was recorded by a high-speed video camera Casio Exilim Pro EX-Fl at a rate of 600 frames per second. Photoperiodically method of determining the quality of fragmentation of the rock mass allowed for a uniform crushing of the rock mass the block. Moreover, the average diameter of the piece in the destruction was $0.56 \mathrm{~m}$, which is $15 \%$ less than in the control area with the traditional arrangement of inclined wells directed perpendicular to the slope of the ledge without taking into account the location of the axis of the well perpendicular to the layers.

\section{Conclusion}

Thus, the nature of the destruction, the intensity and uniformity of fragmentation of layered rocks depend on the direction of force in relation to their deposition. The analysis of the results shows that the proposed method of drilling wells in the rock mass in accordance with the patent for the invention [22] allowed to improve the quality of preparation of rocks for excavation and ultimately increase the productivity of the excavator PH-2300 № 2387 with a bucket capacity of $25 \mathrm{~m} 3$ from 13 to 15 thousand $\mathrm{m} 3$ per shift.

\section{References}

1. M. V. Machinski, The explosive case, 26, 12, (1936)

2. M. V. Machinski, Tech. Phys., 3, 2 (1933)

3. G. I. Pokrovskiy, Explosion (Nedra, Moscow, 1973)

4. G. I. Pokrovskiy, I. S. Fedorov, The effect of impact and explosion in deformable masses (Gosstroiizdat, Moscow, 1957)

5. M. A. Cook, The Science of Industrial Explosives (JDBB, Toronto, 1974) 
6. J. S. Krasinski, A. Khosla, Fluid Mech., 1, 500 (1974)

7. A. N. Khanukayev, Physical Nature of Rock Breakage, Problems of the Theory of Destruction of Rocks by Explosives, (Publishing House of Academy of Science, Moscow, 1958)

8. T. Sakurai, J. Ind. Explosives. Society, 20:1 (1959)

9. J. M. Walsh, R. H. Christian, Phys. Rev, 97 (1954)

10. O. E. Vlasov, The basics of the theory of destruction of rock by explosion: collection: Questions of the theory of destruction of rocks by explosion (Publishing House of Academy of Science, Moscow, 1958)

11. O. E. Vlasov, Basic theories of the explosion. (Publishing House of Academy of Science, Moscow, 1957)

12. O. E. Vlasov, S. A. Smirnov, The basis of the calculation of the crushing of rocks by explosion. (Publishing House of Academy of Science, Moscow, 1962)

13. A. N. Khanukaev, The energy of the stress waves in the destruction of rocks by explosion. (M. Gosgortekhizdat, 1962)

14. E. P. Maksimova, Modeling of the process of explosive destruction. Collection: Mining issues (Publishing House of Academy of Science, Moscow, 1958)

15. F. A. Baum, M. A. Bareges, The explosive case, 52:9 (1962)

16. F. A. Baum, The explosive case, 52:9, 262 (1963)

17. F. A. Baum, K. P. Stanyukovich, B. I. Shekhter, Physics of explosion (Publishing House of Academy of Science, Moscow, 1959)

18. A. F. Belyaev, M. A. Sadovskii, Blast physics (Publishing House of Academy of Science, Moscow, 1952)

19. M. F. Drukovanyi, E. I. Efremov, M. G. Novozhilov, A. A. Tereshchenko, Blasting of high ledges (Publishing House of Academy of Science, Moscow, 1964)

20. G. P. Paramonov, Assessment of the impact fracture of the rock mass for its destruction in the production of blasting (Mining Institute, St.-Petersburg, 2013)

21.N. Ja. Repin, Preparation of rocks for excavation. Part 1: Study guide (Publishing house of Moscow State University, Moscow, 2009) 